\title{
Cosmological tests of gravity
}

\author{
Kazuya Koyama* \\ University of Portsmouth \\ E-mail: Kazuya.Koyama@port.ac.uk
}

\begin{abstract}
The discovery of the accelerated expansion of the Universe has come relatively late in our study of the cosmos, but in showing that gravity can act repulsively, it has opened up many new questions about the nature of gravity and what the Universe might contain. Is the acceleration being driven by dark energy? Or is general relativity (GR) itself in error, requiring a modification at large scales to account for the late acceleration? Structure formation in our Universe can be different even if the geometry of the homogeneous and isotropic universe is the same in these two classes of models, offering a possibility to distinguish between them observationally. Non-linear structure formation is complicated by the fifth force that commonly appears in modified gravity models and new techniques are required to analyse it. We will discuss novel methods to test GR on cosmological scales, building on the recent developments of N-body simulations for modified gravity.
\end{abstract}

Frontiers of Fundamental Physics 14 - FFP14,

15-18 July 2014

Aix Marseille University (AMU) Saint-Charles Campus, Marseille

${ }^{*}$ Speaker. 